\title{
IONIZED GAS AND STELLAR CONTENT \\ IN A SAMPLE OF HII GALAXIES
}

\author{
J.M. Vilchez, J. Cepa, C. Esteban \\ Instituto de Astrofisica de Canarias \\ 38200-La Laguna, Tenerife, Spain
}

\begin{abstract}
We present the first results of an extensive study of the stellar content and the ionized gas structure for a sample of BCGs. Deep broad (BVRI Johnson) and narrow $\left(\mathrm{H}_{\alpha}\right) \mathrm{CCD}$ images have been obtained for about 25 galaxies. For a subset of objects, selected on the basis of their apparent structure, long-slit spectroscopy has also been obtained in order to investigate the interaction between the underlying stellar population and the ionized gas.
\end{abstract}

\section{Introduction}

Blue Compact Galaxies (BCG) are dwarf systems which present vigorous star formation giving rise, for the most recent burts, to several giant HII regions. These giant HII regions generally dominate the optical light of the galaxy, and therefore their emission line spectrum can be easily measurable with intermediate size telescopes. BCG's with such giant HII regions, most of them being rich in $\mathrm{HI}$ gas and oxygen poor, form a substantial subset of the class of objects known as HII Galaxies (Hazard 1985).

The environment where bursts of star formation take place in these galaxies is a matter of considerable interest, because of the importance for the understanding of the evolution of galaxies in general. HII galaxies offer an opportunity to study the very first moments of the evolution of galaxies. In this context, the study of the underlying (young/old) stellar population and its interaction with the observable (ionized) gas, could provide useful information about how star formation proceeds. On the other hand, the knowledge of the nature of the stellar content allows to decide wether there are genuinely young galaxies (in contrast with metal rich but fresh starbursts) and whether the elements present in the ionized gas result from nucleosynthesis in previous generations of stars or just come from the one responsible of the observable HII regions. In order to answer some main questions related to the stellar content in these dwarfs and its interaction with the ionized gas, we carried out a program of combined deep CCD imaging and long-slit spectroscopy for a selected sample of HII galaxies. In the present paper we report some recent results on the photometric properties of the, up to date, studied galaxies; also we present combined spectroscopy and imaging work for several well known dwarfs, and pay especial attention to I $\mathrm{Zw} 18$ (the lowest abundance known HII galaxy).

Several studies of the underlying population in BCG's have been carried out in the last few years making use of CCD cameras capable of reaching very low surface brightness; notably by Loose and Thuan (1985) using B and R filters and by Kunth et 
al. (1985, 1988) using Geneva $B_{1}, B_{2}$, Gunn $R$ and Gunn-Mould I filters. The results appear to show that $\mathrm{BCG}^{6}$ 's present a large variety of morphological types, ranging from more or less smooth circular or elliptical structures easily detectable out to a few Kpc, to cases where nothing is visible beyond the HII region itself. Our CCD observations combine broad (Johnson $\mathrm{B}, \mathrm{V}, \mathrm{R}, \mathrm{I}$ ) and narrow (mainly $\mathrm{H}_{\alpha}$ ) band filters, and have been performed for about 25 galaxies. In addition, long-slit spectroscopy in the range $\lambda \lambda$ 3500-7400 $\AA$ has been obtained for a subset of $\approx 15$ galaxies of the sample, selected mainly on the basis of their observed structure. Since typical dimensions of Giant HII complexes in galaxies are of the order of some 200 pc (e.g. Diaz et al. 1987), our imaging has been performed with good spatial resolution (seeing and pixel sampling) in order to resolve individual star forming areas ( 1 arcsec is equivalent to some $60 \mathrm{pc}$ at 10 $\mathrm{Mpc}$ ). The requiered pixel sampling was such that allowed the Point Spread Function to be well resolved and sampled by a significative number of pixels; this fact is crucial for a correct continuum substraction in the narrow band images. Finally, good seeing conditions, necessary to optimize our instrumental set up, were achieved in most cases. Table $I$ is a journal of the CCD observations, including the telescopes, instrumentation and observing conditions of the program.

The spectroscopic observations were performed using the IDS + IPCS attached to the $2.5 \mathrm{~m}$ INT at the Observatorio del Roque de los Muchachos (La Palma). A dispersion of $2 \AA /$ pixel was used giving a spectral resolution of $\approx 3.5 \AA$ from $3500-7400 \AA$. For each object, a total of 110 spectra were recorded in the spatial direction, one every 1.5 arcsec along the slit. The seeing was oscillating between 0.9 and 1.3 arcsec. The spectral resolution attained was sufficient to resolve underlying absorption features present in some of the galaxies studied (notably in the cases of Mkn 314 and II $\mathrm{Zw} 33$ discussed below), while the spatial sampling allowed to localize these apparently more evolved HII complexes within the galaxies.

The sample of galaxies was selected from the master lists of Thuan and Martin (1981), Thuan (1985) and Longmore et al. (1982), being the more restrictive condition the existence of accurate data of $\mathrm{HI} /$ total masses. Some of our objects are in common with the work of Loose and Thuan (1985), though they have been observed in more bands and with reasonably photometric conditions. A complete description of the galaxy sample and derived properties will be published elsewhere (Vilchez, Cepa, Esteban $1989 \mathrm{~b}$ ). The present paper is intended to present a progress report of some of the basic structural parameters derived for the sample, and the methodology applied, as well as to review some of the results obtained for three particularly interesting galaxies: Mkn 314, II Zw 33 and, especially, I Zw 18.

TABLE I.- Journal of CCD Observations

\begin{tabular}{llll}
\hline Telescope & Instrument & Resolution & Mean Seeing \\
\hline WHT $4.2 \mathrm{~m}$ & Cass. TaurusII Cam. $0.26 \% / \mathrm{pix}$ & $0.7-1.2 \%$ \\
CAHA $3.5 \mathrm{~m}$ & Prime CCD Cam. & $0.50 \% / \mathrm{pix}$ & $2 \%$ \\
INT $2.5 \mathrm{~m}$ & Prime CCD Cam. & $0.70 \% / \mathrm{pix}$ & $1-1.6 "$ \\
JKT $1.0 \mathrm{~m}$ & Cass. CCD Cam. & $0.50 " / \mathrm{pix}$ & $1-1.4 \%$ \\
\hline
\end{tabular}




\section{Global Structural Parameters}

Total fluxes and colours have been derived for the program galaxies. Total fluxes were obtained integrating the flux in all the pixels with signal above the $3 \sigma$ level, generally corresponding to $26.5 \mathrm{mag} / \operatorname{arcsec}^{2}$, (previously, the CCD frames were masked to avoid stars in the field, hot spots, etc.). As an inmediate consequence the derived total luminosities are far more reliable than those obtained by means of aperture photometry and/or magnitude limited observations. Figure 1 (a) shows the correlation between the derived absolute magnitudes in the blue $\mathrm{M}_{B},\left(\mathrm{H}_{o}=75\right.$ assumed $)$, and the abundance of oxygen $(12+\log \mathrm{O} / \mathrm{H})$ for the galaxies in the program with known $\mathrm{O} / \mathrm{H}$ from the literature and/or our spectroscopy. In Figure 1 (b) the relationship between HI mass and the derived $\mathrm{M}_{B}$ is presented. HI masses have been taken from Thuan and Martin (1981), Brinks and Klein (1985) and Combes (1985). A strong correlation is present in both figures in the sense that lower abundance galaxies seem to have larger blue magnitudes, whilst larger HI masses are associated to brighter galaxies. The brightest galaxy studied appears to be III $\mathrm{Zw} 102$, with an absolute magnitude more adecuated to that of a giant galaxy (unless there is something wrong with the distance modulus quoted in the literature (Thuan and Martin, 1981). This suggestion could be consistent with the very high dust content found for this galaxy (the galaxy presents very clear dust lanes in our colour images), a feature which appears to be not very common among low abundance compact HII galaxies; in addition, the high $\mathrm{O} / \mathrm{H}$ value that is indicated by its IPCS spectra points to the same direction. Overall, the relationship between $\mathrm{M}_{B}$ and oxygen abundance found by Skillman et al. (1989) for his compilation of Dwarf Irregulars, $12+\log \mathrm{O} / \mathrm{H}=-0.15 \mathrm{M}_{B}+5.5$, appears to be very well followed by the program galaxies, from the "metallicity" of $\mathrm{I} Z \mathrm{w} 18\left(\mathrm{M}_{B}=-14\right)$ up to the one of the giant III Zw 102.
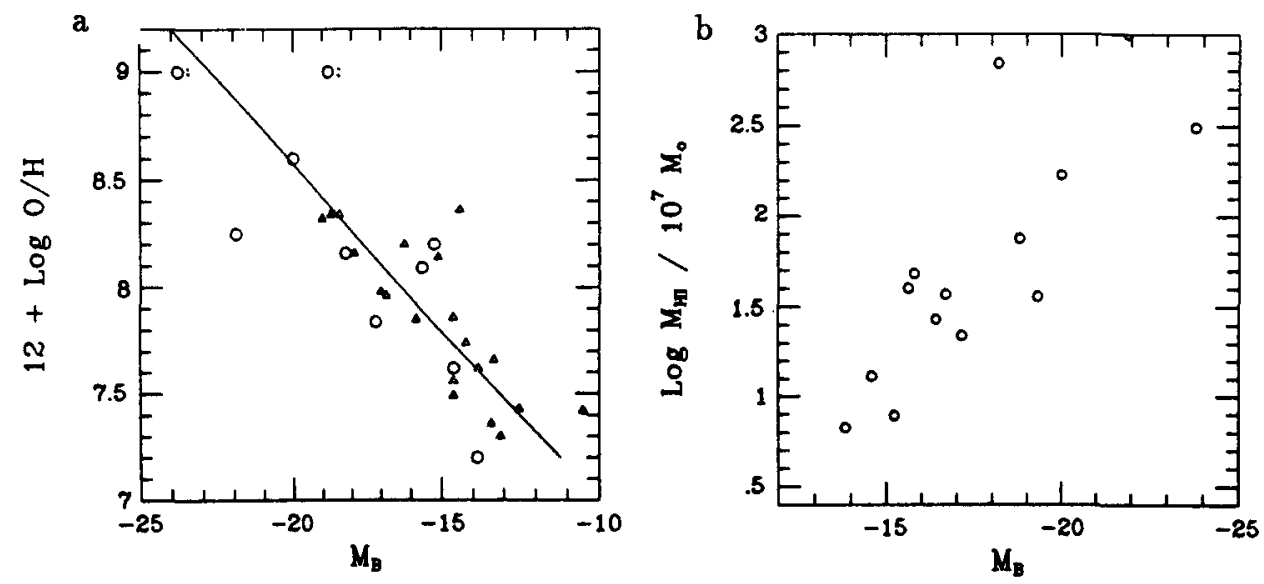

Figure 1.- The relationship between the oxygen abundance, a), and HI mass, b), with the derived $M_{B}$ magnitude. In a) the triangles are objects from Skillman et al. (1989). 
In order to make a systematic analysis of structural parameters of the galaxies, independently of their particular apparent morphology, we are investigating the extension of the formalism developed for elliptical/spiral galaxies to these type of galaxies; these show such a rich variety of morphologies that systematical studies of their structure are very rare. We have extended the concept of "effective radius" to this type of galaxies by means of the definition of an "equivalent effective area", which is the galactic area enclosed within the isophote that includes the half of the total luminosity of the galaxy. Also, an "equivalent effective radius" can then be defined, formally, as the one of a circle with area equal to the "equivalent effective area"; likewise, an "equivalent surface brightness" profile can be derived plotting the surface brightness at every isophote versus its corresponding "equivalent radius". The basic equations used to extend this formalism can be expressed as follows:

$$
\frac{1}{2} F_{\text {Tot }}^{\lambda}=\int_{S B_{\text {efs }}}^{S B_{\max }} f_{\lambda}(x, y) d x d y
$$

where $F_{\text {Tot }}^{\lambda}$ is the total flux of the galaxy in the band $\lambda, f_{\lambda}(x, y)$ is the measured flux in the pixel $(x, y)$ in the band $\lambda$ and $S B_{e f f}$ and $S B_{\max }$ are the surface brightnesses of the isophote enclosing the "equivalent effective area" and the maximun surface brightness observed in the galaxy, respectively, both referred to the band $\lambda$. And similarly, the "equivalent effective area" can then be defined by:

$$
A_{e f f}=\int_{S B_{e f f}}^{S B_{\text {max }}} d x d y=\pi R_{e f f}^{2}
$$

Following this method it should be possible to distinguish, for instance, between Low Surface Brightness and Very Compact Galaxies, since they should present different relative fractions of the total surface per unit interval of surface brightness. A detailed application to the program galaxies is in course.

\section{Selected Examples: the cases of II Zw 33, Mkn 314 and I Zw 18}

In the following we present, as an example, some preliminary results for three individual galaxies choosed on the basis of their particular structure. First, the galaxies Mkn 314 and II Zw 33 show a striking morphology in our broad band frames, characterized by the existence of twisted isophotes and the presence of aligned knots of star formation. This morphology has been interpreted, in the case of Mkn 314 (e.g. Loose and Thuan 1985), as the possible relic of a merging process; although the HI velocity pattern for Mkn 314 is highly turbulent, in contrast with the ordered motions observed in the merging BCG II Zw 40 (Brinks and Klein, 1985, 1988).

On the other hand, on the basis of CCD imaging, it has been claimed that II $\mathrm{Zw}$ 33 should be a barred spiral galaxy (Loose and Thuan 1985), a suggestion consistent with recent findings indicating that II $\mathrm{Zw} 33$ is an old object (Vilchez, Cepa, Esteban 
1989a). Our spectroscopy shows that certainly the giant HII complexes in II Zw 33 seem to be more chemically evolved, as a whole, than the ones in Mkn 314, which presents an oxygen abundance at least a factor three lower $(12+\log \mathrm{O} / \mathrm{H}=8.15)$. On the other hand, II $\mathrm{Zw} 33$ shows clear evolutionary differences between the starburst in the centre of the galaxy, and the HII knots apparent also in broad band images; the central regions are more evolved as judged from their equivalent width of $H \beta$, and the presence of strong (broad) absorption features in the underlying continuum. Figure 2 illustrates the relative behaviour of the equivalent width of $\mathrm{H} \beta$ in II $\mathrm{Zw} 33$, from the comparison of the spatial profiles of $\mathrm{H} \beta$ and the Continuum along the slit.
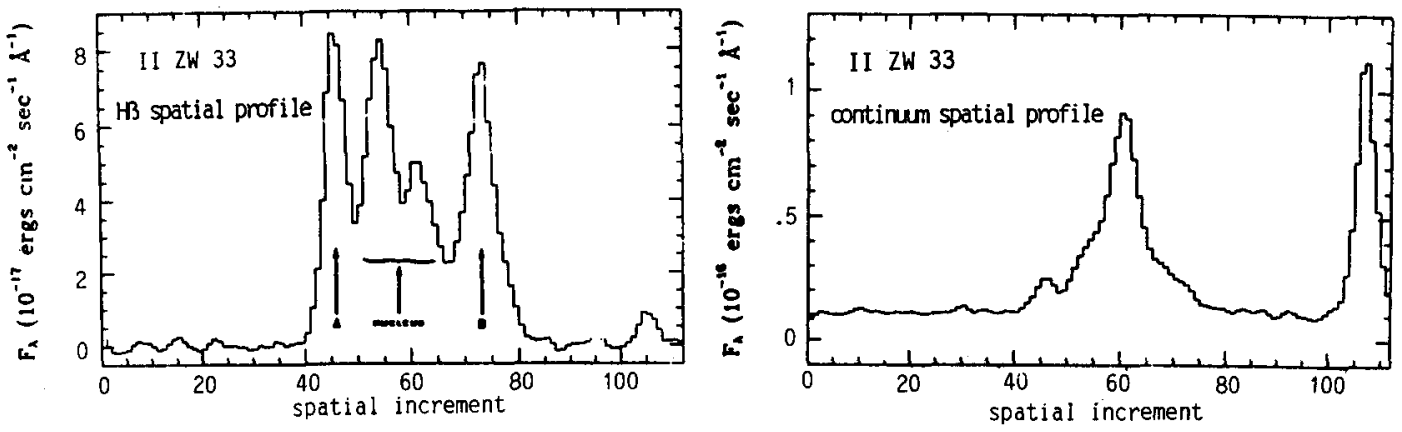

Figure 2 Relative Behaviour of the $H \beta$ (left) and Continuum (right) profiles in II $Z w 99$

Perhaps the most interesting case among BCG's is the one of I $\mathrm{Zw} 18$, because of its extreme abundance, $\mathrm{O} / \mathrm{H}=1 / 50$ solar. This characteristic has lead to the suggestion that the observed starburts in the galaxy constitute the very first star formation in $\mathrm{I} \mathrm{Zw}$ 18 , and the observed abundance ratios have been interpreted in terms of nucleosynthesis in the current burst (Kunth and Sargent 1986). Our observations have revealed the presence of a substantial amount of stellar population having colours typical of disc stars of some $10^{9}$ years; suggesting that the current burst is perhaps the first important burst of star formation but definitively not the first star formation in the galaxy (Vilchez, Cepa, Pagel 1989). Therefore $I \mathrm{Zw} 18$ is not a genuinely young galaxy (in the sense pointed out in section 1 above). In addition, a set of around 20 new non stellar sources in the field of $\mathrm{I} \mathrm{Zw} \mathrm{18,} \mathrm{have} \mathrm{been} \mathrm{catalogued} \mathrm{and} \mathrm{measured.} \mathrm{All} \mathrm{the} \mathrm{sources} \mathrm{are} \mathrm{well}$ above the $3 \sigma$ level in the $\mathrm{R}$ frame, and present non stellar well resolved profiles. Most of them we believe are actually related to $\mathrm{I} Z \mathrm{w} 18$, since the search was performed within an area roughly defined by the $\mathrm{HI}$ map of Lequeux and Viallefond (1980). Figure 3 shows the colour-colour diagram for the measured sources, with colours ranging from those typical of $\mathrm{OB}$ to $\mathrm{KM}$ stars. Finally, the photometry obtained for I $\mathrm{Zw} 18$ has allowed us to compute an approximate mass in stars which in turns, when compared to the total mass of HI (e.g. Viallefond et al. 1987), has revealed that the prediction of the Simple Model of chemical evolution is not far from the locus of $\mathrm{I} \mathrm{Zw} 18$. Therefore, the outstanding difference between "dynamical" and "chemical" masses can be telling us that a substantial amount of dark matter is probably present. 
Figure 3.- Colour-Colour diagram of the set of non-stellar sources detected in the field of I $Z w 18$.

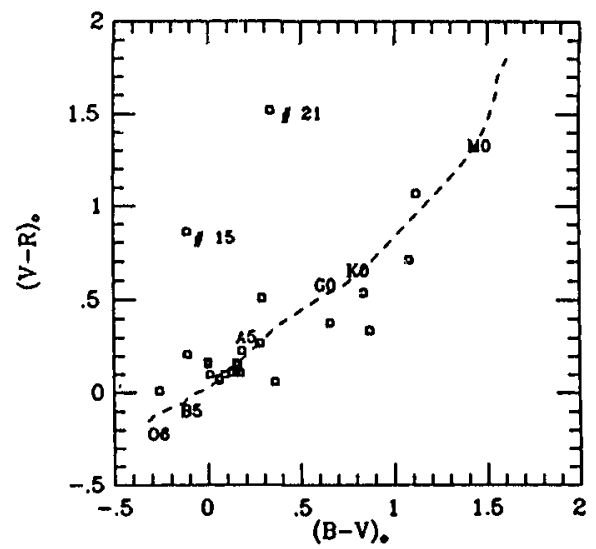

References

Brinks, E., Klein, U., 1985. in Star-Forming Dwarf Galaxies and Related Objects. D. Kunth, T.X. Thuan, J.T. Thanh Van, eds., p. 281. Frontieres. Paris

- 1988. Mon. Not Roy. astr. Soc., 231, 63P.

Combes, F., 1985. in Star-Forming Dwarf Galaxies and Related Objects. D. Kunth, T.X. Thuan, J.T. Thanh Van, eds., p. 307. Frontieres. Paris

Díaz, A., Terlevich, E., Pagel, B.E.J., Vilchez, J.M., Edmunds, M.G.,1987. Mon.

Not. Roy. astr. Soc., 226, 19.

Hazard, C., 1985. in Star-Forming Dwarf Galaxies and Related Objects. D. Kunth, T.X. Thuan, J.T. Thanh Van, eds., p. 9. Frontieres. Paris.

Kunth, D., Martin, J.M., Maurogordato, S., Vigroux, L., 1985. in Star-Forming Dwarf Galaxies and Related Objects. D. Kunth, T.X. Thuan, J.T. Thanh Van, eds., p. 89. Frontieres. Paris.

Kunth, D., Maurogordato, S., Vigroux, L., 1988. Astron. Astrophys., 204, 10.

Kunth, D., Sargent, W.L.W., 1986. Astrophys. J., 300, 496.

lequeux, J., Viallefond, F., 1980. Astron. Astrophys., 91, 269.

Longmore, A.J., Hawarden, T.G., Goss, W.M., Mebold, U., Webster, B..L., 1982. Mon. Not Roy. astr. Soc., 200, 325.

Loose, H.H., Thuan, T.X., 1985. in Star-Forming Dwarf Galaxies and Related Objects. D. Kunth, T.X. Thuan, J.T. Thanh Van, eds., p. 73. Frontieres. Paris

Skillman, E.D., Kennicut, R.C., Hodge, P., 1989. Preprint.

Thuan, T.X., 1985. Astrophys. J., 299, 881.

Thuan, T.X., Martin, G.E., 1981. Astrophys. J., 247, 823.

Viallefond, F., Lequeux, J., Comte, G., 1987. in Starbursts and Galaxy Evolution. T. Montmerle, J.T.T. Van, eds., p. 139. Frontieres. Paris.

Vilchez, J.M., Cepa, J., Esteban, C., 1989a. in $2^{\text {nd }}$ Tex-Mex Conference on Astrophysics. In press.

Vilchez, J.M., Cepa, J., Esteban, C., 1989b. In prep.

Vilchez, J.M., Cepa, J., Pagel, B.E.J., 1989. Preprint.

\section{Discussion:}

PAGEL (Comment): I am delighted to see your relation between abundance and ratio of mass of gas to mass of gas plus stars. The discrepancies with total mass based on the virial theorem may raise from dark matter which takes no part in the chemical evolution of visible material. 